\title{
Interband Cascade Infrared Photodetectors with Long and Very-Long Cutoff Wavelengths
}

\author{
Hossein Lotfi ${ }^{1}, \mathrm{Lu} \mathrm{Li}^{1}$, Hao Ye ${ }^{1}$, Robert T. Hinkey ${ }^{1,2}$, Lin Lei ${ }^{1,2}$, Rui Q. Yang ${ }^{1}$, Joel C. \\ Keay $^{2}$, Tetsuya D. Mishima ${ }^{2}$, Michael B. Santos ${ }^{2}$, and Matthew B. Johnson ${ }^{2}$ \\ ${ }^{1}$ School of Electrical and Computer Engineering, University of Oklahoma, Norman, OK, USA \\ ${ }^{2}$ Homer L. Dodge Department of Physics and Astronomy, University of Oklahoma, Norman, \\ OK, USA
}

•Email: $\underline{\text { hossein.lotfi@ou.edu; ‘ Email: Rui.Q.Yang@,ou.edu }}$

\begin{abstract}
We report our recent studies of interband cascade infrared photodetectors (ICIPs) with 100\% cutoff wavelengths of $16.0 \mu \mathrm{m}$ and $9.2 \mu \mathrm{m}$ at $78 \mathrm{~K}$. The very-long-wavelength infrared (VLWIR) detectors were able to operate at temperatures up to $143 \mathrm{~K}$. Relatively high dark current densities were observed from these VLWIR detectors, which were correlated to the very narrow bandgap and possibly to defects in the materials. These VLWIR detectors also exhibited strongly bias dependent photo-responses, indicating that further research and device optimization are necessary. On the other hand, two-stage ICIPs in the long-wavelength infrared (LWIR) spectrum $(8-12 \mu \mathrm{m})$ were able to operate at higher temperatures (up to $250 \mathrm{~K}$ ) with an extended cutoff wavelength of $\sim 12 \mu \mathrm{m}$. At $78 \mathrm{~K}$, these LWIR detectors showed a bias independent photocurrent with $R_{0} A$ of $115 \Omega . \mathrm{cm}^{2}$, corresponding to a Johnson-noise-limited $D^{*}$ of $4.4 \times 10^{10}$ $\mathrm{cm} . \mathrm{Hz}^{1 / 2} / \mathrm{W}$ at $8.0 \mu \mathrm{m}$.
\end{abstract}


Keywords: III-V semiconductors, Infrared detector, Interband cascade, LWIR, Type-II superlattice, VLWIR.

\section{INTRODUCTION}

Theoretical calculations [1-2] and experimental results [3-4] indicate that type-II InAs/ $\mathrm{Ga}(\mathrm{In}) \mathrm{Sb}$ superlattice (SL) photodetectors are a promising alternative to mercury cadmium telluride (MCT) detectors, especially in the long-wavelength infrared $(8-12 \mu \mathrm{m})$ and very-longwavelength infrared (12-30 $\mu \mathrm{m})$ regions. For this SL material, the absorption coefficient near the band-edge is reduced as the cutoff wavelength of the material is made longer. Thus, in order to achieve a high absorption quantum efficiency with a LWIR SL detector, the absorber must be thicker than those typically used for mid-wavelength infrared (MWIR) detectors. In addition, the carrier lifetime is expected to be lower for narrower-bandgap materials. This implies that narrower-bandgap materials will have shorter carrier diffusion lengths and consequently the attainable quantum efficiency will be lower. The effect could become more detrimental at high temperatures as the diffusion length is further reduced, as suggested by data from the literature [5]. Therefore, a thick SL absorber may not necessarily enhance the external quantum efficiency for a type-II SL detector, especially at high temperatures.

This issue can be circumvented in interband cascade infrared photodetectors (ICIPs) where a discrete multiple absorber architecture is used to increase the collection efficiency of photogenerated carriers and reduce the detector noise [6-11]. In this type of infrared photodetector, an individual absorber is made of an InAs/GaSb type-II SL and sandwiched between hole and electron barriers to form one cascade stage. The electron barrier is made of $\mathrm{GaSb} / \mathrm{AlSb}$ quantum wells (QWs) and the hole barrier is composed of InAs/AlSb QWs. The

placement of the electron and hole barriers on opposite sides of the absorber ensures that 
electrons and holes flowing in opposite directions, making it possible that properly designed ICIPs can be operated as photovoltaic detectors. Experimental investigations of ICIPs in the midwavelength infrared region have demonstrated that they are able to operate at very high temperatures [12-13]. It is expected that the interband cascade approach will have prominent advantages for LWIR and VLWIR detectors, especially for high-operating-temperature (HOT) applications. In this paper, we report our preliminary studies of these ICIPs in the LWIR and VLWIR spectral regions.

This paper is organized as follows: Section 2 discusses the material and fabrication process for both LWIR and VLWIR ICIPs. Section 3 presents the electrical and optical characterization results and discussions for these photodetectors. Finally, Section 4 consists of our concluding remarks with some comments and a perspective for the future research on ICIPs.

\section{DEVICE STRUCTURE, GROWTH AND FABRICATION}

A set of three ICIP structures was designed to target the VLWIR region. For this set, each SL period is composed of 45 - $\dot{A}$-thick InAs and 21.2- $\dot{A}$-thick GaSb layers, with a thin InSb (4.8 $\dot{\mathrm{A}})$ layer inserted into the GaSb-on-InAs layers as an interface to compensate the tensile strain derived from the relatively thick InAs layer. The two- and three-stage devices have 568-nm and 639-nm-thick absorbers. The three-stage detectors have an additional stage with a 752.6-nmthick absorber. The deeper absorbers in these ICIPs are made thicker in order to achieve currentmatching. For comparison, a one-stage device was also examined in the study. The absorber thickness was set as $1.96 \mu \mathrm{m}$, equal to the total thickness of the absorbers in the three-stage design. In order to make electrons the minority carriers, half of the GaSb layers in the SL 
absorbers were $p$-doped with a doping density of $3.9 \times 10^{16} \mathrm{~cm}^{-3}$. The electron and hole barriers in each of these devices have identical designs.

All the ICIP structures reported in this paper were grown by molecular beam epitaxy (MBE). After the structure growth, square mesa devices with edge lengths ranging from 200 to $1000 \mu \mathrm{m}$ were fabricated by using conventional contact UV lithography and wet etching. For VLWIR structures a 228-nm-thick $\mathrm{Si}_{3} \mathrm{~N}_{4}$ layer was used as a passivation layer. The top and bottom contacts consist of sputtered 30-nm-thick Ti and 300-nm-thick Au layers for both VLWIR and LWIR structures.

\section{MEAUREMENTS AND DISCUSSION}

\section{3-1. Very-Long-Wavelength ICIPs}

In this set of ICIPs, the stages were designed with the hole barrier on the top and the electron barrier at the bottom. This is a reversal of our previous stage designs, which had the electron barrier on the top [7-9]. With this design, electrons flow to the top (i.e. away from the substrate) through the hole barrier to the next stage or the contact. This configuration is advantageous for detectors with $p$-type absorbers when the device is under light illumination from the top, because most of the photogenerated minority carriers (i.e. electrons) are closer to the collection point.

Since the hole barrier is at the top of the stage, a positive polarity at the top of the device is equivalent to the operation of a photodiode under a reverse bias. Dark current density $\left(J_{d}\right) v s$. bias voltage $(V)$ for representative one-, two- and three-stage devices at various temperatures $(T)$ is shown in Fig. 1. As displayed in this figure, the dark current density at $78 \mathrm{~K}$ for a one-stage device (e.g. $0.1 \mathrm{~A} / \mathrm{cm}^{2}$ at $50 \mathrm{mV}$ ) was the highest among all three detectors. This is expected based on the theory for an ideal diffusion-limited detector. However, the dark current data for 
some two- and three-stage devices, especially at low temperatures, did not follow what was expected from theoretical predictions $[8,11]$. This was likely due to variations in material uniformity and device fabrication. For devices shown in Fig. 1, at $50 \mathrm{mV}$ and at $78 \mathrm{~K}$, the dark current density was $25 \mathrm{~mA} / \mathrm{cm}^{2}$ for a three-stage device, which was comparable to the corresponding value of $24 \mathrm{~mA} / \mathrm{cm}^{2}$ for a two-stage detector. For a multiple stage detector with diffusion limited dark current, the product of total device resistance $R_{0}$ at zero bias and device area (A) can be written as [11]

$$
R_{0} A=\frac{k_{b} T}{e^{2} g_{t h} L_{n}} \sum_{m} \frac{1}{\tanh \left(\frac{d_{m}}{L_{n}}\right)},
$$

where $g_{\text {th }}$ is thermal generation rate, $e$ is the electron charge, $k_{b}$ is the Boltzmann constant, $m$ denotes the index of stages in a multi-stage detector, $d_{m}$ and $L_{n}$ are the absorber thickness in the $m^{\text {th }}$ stage and the minority carrier diffusion length, respectively. From Eq. (1), one can see that $R_{0} \mathrm{~A}$ is proportional to the sum of $1 / \tanh \left(d_{m} / L_{n}\right)$. The $R_{o} A$ value will be higher for detectors with more stages, and lower for detectors with longer absorbers. Applying this equation to VLWIR detectors at $78 \mathrm{~K}$ and assuming that the minority carrier diffusion length is much longer than the absorber thickness (i.e. $d_{m} / L_{n}<<1$ ), the $R_{0} A$ for two- and three-stage detectors should be 6.5 and 9.1 times larger than for the one-stage detector, respectively. The experimentally extracted values of the $R_{0} A$ for the two- and three-stage detectors were $2.4 \Omega . \mathrm{cm}^{2}$ and $2.3 \Omega . \mathrm{cm}^{2}$, which are about 4 times higher than that of the one-stage device $\left(0.56 \Omega . \mathrm{cm}^{2}\right)$. Hence, $R_{0} A$ for multiplestage devices was not as high as expected from Eq. (1).

This discrepancy indicates that dark current in these detectors deviated from a diffusion limited behavior, as mentioned above, and that the carrier transport could be affected by other factors so 
that the minority carrier diffusion length might be shorter than the absorber thickness. At temperatures above $100 \mathrm{~K}$ the dark current density was lower for the three-stage detector than for the two-stage device, which is qualitatively more consistent with what is implied in Eq. (1). The irregular behavior at low temperatures suggests that additional factors (such as variations in the material and fabrication quality) influence the dark current in these devices. For all devices from the three wafers, the dark current was sensitive to a bias voltage across the entire range of applied reverse bias and did not saturate. Dark current densities in VLWIR detectors are intrinsically high due to the very narrow bandgap. Additionally crystalline defects (surface defect density of $5-10 \times 10^{5} \mathrm{~cm}^{-2}$ ) and defect-assisted tunneling open additional channels that increase the dark current in these devices.

Optical response measurements were performed using a calibrated blackbody placed at a distance of $9.5 \mathrm{~cm}$ from the device under test (DUT). The blackbody aperture radius was set to $0.76 \mathrm{~cm}$ and a Nicolet 8700 FTIR spectrometer was used to collect the device relative response spectrum. Unlike our previous devices, which had a strong photo-response at zero-bias [10], the devices in this set required an applied bias in order to observe a substantial photocurrent. This suggests some non-idealities in the carrier transport. One possibility is that an unintentional electrostatic barrier was formed between the absorber and the hole barrier, which impeded the collection of the photo-generated electrons. Another possibility is that the SL absorber may have become $n$-type, despite the intentional introduction of $p$-dopants during the growth process. This is possible because the SL absorber is InAs rich (about twice as much InAs as GaSb in the SL for the VLWIR detectors) and the background doping of MBE-grown InAs is $n$-type. Also, for VLWIR detectors, the very narrow bandgap of the absorber makes it more likely that the intrinsic carrier concentration due to thermal excitation is higher than the dopant concentration. 
Thus, even with intentional $p$-doping of the absorber, the SL absorber may be $n$-type for VLWIR detectors, even at low temperatures [14]. This implies the diffusion length could be shorter than expected, due to the low vertical hole mobility in InAs/GaSb SLs. Consequently, the collection of photogenerated carriers would be less efficient, particularly in the flipped ICIPs, where most holes are generated far from the collection point. In this case, the applied reverse bias would accelerate holes towards the electron barrier, i.e. the collection point.

The responsivity spectra for a two-stage detector at 78 and $100 \mathrm{~K}$ are shown in Fig. 2. At $10 \mu \mathrm{m}$ the responsivity was $0.48 \mathrm{~A} / \mathrm{W}$ at $78 \mathrm{~K}$ under $300 \mathrm{mV}$ bias. However, the responsivity was low at zero bias for all tested devices made from the three VLWIR detector wafers. As shown in inset (a) to Fig. 2, for the zero-bias responsivity at $\lambda=10 \mu \mathrm{m}$, the single-stage device with the thickest absorber had the lowest value, while the three-stage device, with the second thickest absorber in the third stage, had the second lowest value. Their zero-bias responsivity increased with temperature in the range of 78-143 K. Such a temperature dependence characteristic was observed for all tested devices made from the three wafers. This observation supports the existence of an unwanted electrostatic barrier that blocks the collection of photogenerated carriers. The influence of this barrier was less detrimental at higher device temperatures. At 78 and $100 \mathrm{~K}$, the photoresponse of these detectors increased significantly with reverse bias. For example, the responsivity at $10 \mu \mathrm{m}$ increased from $5.3 \mathrm{~mA} / \mathrm{W}$ at zero bias to $480 \mathrm{~mA} / \mathrm{W}$ at 300 $\mathrm{mV}$ for a two-stage ICIP at $78 \mathrm{~K}$. At $100 \mathrm{~K}$, the responsivities were reduced compared to the values for the three devices at $78 \mathrm{~K}$ and at the same bias voltage. For example, under $150 \mathrm{mV}$ the one-stage device exhibited a significant $(\sim 49 \%)$ reduction in its photo-response as the temperature was increased from 78 to $100 \mathrm{~K}$. However, the responsivities for the two- and threestage devices at the same bias $(150 \mathrm{mV})$ were moderately decreased $(6 \%$ for two-stage and $14 \%$ 
for three-stage) compared to their values at $78 \mathrm{~K}$. This is indicative of poorer collection of photogenerated carriers in thick-absorber detectors at higher temperatures.

The responsivity for temperatures above $100 \mathrm{~K}$ for the two-stage detector (under zero-bias) is shown in inset (b) to Fig. 2. We attempted to identify the maximum operating temperature at which a meaningful response spectrum could be obtained from these detectors using the FTIR spectrometer. Although the measurements were noisy, both two- and three-stage detectors were able to operate up to $185 \mathrm{~K}$, which is higher than the maximum operating temperature of $167 \mathrm{~K}$ for the single absorber detector.

To make a further performance comparison of the devices made from the three VLWIR ICIP wafers, the normalized detectivity, $D^{*}$, at $78 \mathrm{~K}$ is shown in Fig. 3. As the maximum photoresponse was attained under a reverse bias, the shot noise due to dark current is included in the $D^{*}$ evaluation:

$$
D^{*}=\frac{R_{\lambda}}{\sqrt{\frac{4 k_{B} T}{R A}+2 q J}} .
$$

As shown in Fig. 3 the value of $D^{*}$ for the two-stage detector was comparable to that for the onestage detector, while it was significantly lower in the three-stage detectors. This is related to the combined effects of their responsivities and dark currents as implied by Eq. (2). In the one-stage device, the responsivity is substantially higher because of the thicker absorber $(1.96 \mu \mathrm{m})$. For example, at a bias of $150 \mathrm{mV}$ and $\lambda=10 \mu \mathrm{m}$ the responsivity was $0.93 \mathrm{~A} / \mathrm{W}$ for one-stage detector; while it was 0.4 and $0.17 \mathrm{~A} / \mathrm{W}$ for two- and three-stage detectors. As the two- and three-stage detectors were both designed to be current-matched, their responsivities were expected to be equal. But the three-stage detectors had the lower responsivity, which could be 
due to imperfect current matching and the requirement of a higher bias voltage for reaching the maximum photocurrent with an additional stage. The low responsivity, combined with the higher-than-expected dark current, resulted in the lower $D^{*}$ observed in three-stage detectors.

Under a $150 \mathrm{mV}$ bias, the $D^{*}$ for the single absorber detector at $\mathrm{T}=78 \mathrm{~K}$ and $\lambda=10 \mu \mathrm{m}$ was $2 \times 10^{9}$ $\mathrm{cm} . \mathrm{Hz}^{1 / 2} / \mathrm{W}$, which is lower than $D^{*}=4 \times 10^{10} \mathrm{~cm} \cdot \mathrm{Hz}^{1 / 2} / \mathrm{W}$ reported for a $14-\mu \mathrm{m}$-cutoff-wavelength detector with a similar absorber thickness $(1.9 \mu \mathrm{m})[15]$. The peak responsivity of $1.1 \mathrm{~A} / \mathrm{W}$ obtained for the single stage detectors was comparable to the peak responsivity of $1.4 \mathrm{~A} / \mathrm{W}$ at similar wavelengths reported in Ref. 15 . This implies that the lower $D^{*}$ in our detectors was due to the higher levels of noise. If the maximum photo-response could be realized under zero-bias, the corresponding Johnson-noise-limited $D^{*}$ would be $1.3 \times 10^{10} \mathrm{~cm} \cdot \mathrm{Hz}^{1 / 2} / \mathrm{W}, 1.1 \times 10^{10}$ $\mathrm{cm} . \mathrm{Hz}^{1 / 2} / \mathrm{W}$, and $5.6 \times 10^{9} \mathrm{~cm} . \mathrm{Hz}^{1 / 2} / \mathrm{W}$ for the one-stage, two-stage, and three-stage detectors, which is more than 6 times higher than the corresponding maximum values of $D^{*}$ obtained for these devices under a bias. This means that much higher levels of $D^{*}$ can be achieved by merely optimizing the structure for a bias-independent photoresponse.

We note that in the results presented above, the voltage applied was not sufficient to reach full response. In an ideal case where the absorber thicknesses are shorter than the carriers' diffusion length the particle conversion efficiency (PCE), which is defined as the total number of photogenerated carriers that are collected in any stage, is proportional to the total absorber thicknesses. Hence the PCE should be equal in both one- and three-stage detectors due to their identical total absorber thicknesses. However, the experimental extracted value of PCE was smaller (about $70 \%$ at $10 \mu \mathrm{m}$ ) than that in the one-stage device, which is indicative of some level of current mismatch. Another factor was the bias voltages that we were able to apply were not sufficient to reach the maximum photocurrent. As the photoresponse exhibited a very strong 
bias dependence, higher bias might be required for a detector with more stages to reach its photocurrent saturation. The high levels of dark current and instrument limitations precluded measurements outside a small range of voltages near zero-bias. This also could affect the maximum $D^{*}$. At higher temperatures $(100-143 \mathrm{~K})$ the highest $D^{*}$ was obtained for the twostage ICIP, however this result may have been constrained by the limited bias voltage that could be applied to the three different VLWIR detectors.

\subsection{Long-Wavelength ICIPs}

An additional set of two ICIP structures with two stages (wafers R120 and R121) were designed for LWIR operation. The layer sequences for the two wafers were nearly identical, aside from their InSb strain-balancing layers. The absorber layers had thicknesses of $620.0 \mathrm{~nm}$ and 756.4 $\mathrm{nm}$, with each SL period composed of $36.3 \dot{\mathrm{A}}$ of InAs and $21.9 \dot{\mathrm{A}}$ of GaSb. In each SL period of R120, a 1.9- $\dot{A}$-thick InSb layer was intentionally inserted into both the InAs-on-GaSb and the GaSb-on-InAs layers as the interface layer. However, in R121, the InSb layer (3.8 $\dot{\mathrm{A}})$ is only inserted into the GaSb-on-InAs layers. Similar to VLWIR ICIPs the absorber layers are partially $p$-doped (half of the total absorber thickness) at $3.5 \times 10^{16} \mathrm{~cm}^{-3}$ in the GaSb layers. The device fabrication process followed the same steps as for the above-mentioned VLWIR detectors except the passivation layer: a two-layer passivation consisting of $170 \mathrm{~nm}$ of $\mathrm{Si}_{3} \mathrm{~N}_{4}$ followed by $137 \mathrm{~nm}$ of $\mathrm{SiO}_{2}$ was used for these detectors.

For dark current measurements the devices were mounted in a cryostat with a cold shield. Many devices made from the two wafers were tested. At $78 \mathrm{~K}$, the average $R_{0} A$ for the $\mathrm{R} 121$ wafer (16 detectors) was $58.3 \Omega . \mathrm{cm}^{2}$ which was higher than the average value $\left(32.7 \Omega . \mathrm{cm}^{2}\right)$ for devices (17 
detectors) made from the R120 wafer. Comparing the best performing devices from both wafers (Fig. 4), the dark current at $50 \mathrm{mV}$ was $3.0 \times 10^{-4}\left(3.5 \times 10^{-4}\right) \mathrm{A} / \mathrm{cm}^{2}$ for R121 (R120) with corresponding $R_{0} A$ of 150 (115) $\Omega . \mathrm{cm}^{2}$ at $78 \mathrm{~K}$. The dark current characteristics for these two wafers did not exhibit any improvement for the two InSb interface detectors (R120) over the one InSb interface detectors (R121), which is in agreement with our preliminary material characterizations (X-ray diffraction and optical microscopy). This suggested that wafers R120 and R121 had comparable material quality. The dark current densities for the LWIR devices were much lower than those observed in the VLWIR devices, due to the shorter cutoff wavelength, improved device fabrication and lower surface defect densities $\left(<1 \times 10^{4} \mathrm{~cm}^{-2} v s\right.$. $\sim 1 \times 10^{6} \mathrm{~cm}^{-2}$ as identified by Nomarski microscopy).

Dark current values at different operating temperatures for the R120 wafer are shown in Fig. 5. In order to identify the dominant dark current mechanism, an Arrhenius dark current plot (at 50 $\mathrm{mV}$ bias) across the $100-250 \mathrm{~K}$ temperature range was fit to the following equation:

$$
J \propto C e^{-\frac{E_{a}}{k_{B} T}}
$$

where $E_{a}$ is the activation energy. As shown in the inset to Fig. 5, the activation energy is estimated to be $\sim 102 \mathrm{meV}$. The activation energy (not shown in the figure) for devices from wafer R121 is $126 \mathrm{meV}$. At $78 \mathrm{~K}$ the corresponding bandgap energy at the $50 \%$ cutoff response was 144 and $148 \mathrm{meV}$ for wafers R121 and R120, respectively. This means the fitted activation energy is closer (yet lower) to the device bandgap for wafer R121. These activation energies imply that the detectors are neither diffusion limited nor dominated by the G-R process (activation energy is larger than $E_{\mathrm{g}} / 2$ ) for this temperature range. The deviation from the diffusion limit is probably related to the non-uniform doping that is applied to the absorber 
regions, which may create an undesired electric field in the absorber regions with the potential to deteriorate the G-R process in the absorber layers. Further investigations are ongoing to quantify the competing dark current mechanisms in ICIP structures.

The optical response characterization of the devices was performed using a $600 \mathrm{~K}$ blackbody source with a $0.76 \mathrm{~cm}$ aperture size positioned $30 \mathrm{~cm}$ away from the DUT. The devices from R120 showed no bias-dependence in the response up to $167 \mathrm{~K}$. However, at higher temperatures (as shown in Fig. 6), an applied bias was required in order to achieve full collection of the photogenerated carriers. At these higher device temperatures, a higher reverse bias was required to reach saturation. The zero-bias response spectra at temperatures of 240 and $250 \mathrm{~K}$ are also shown in the inset to Fig. 6. For these temperatures, we were not able to apply a sufficient bias to the detectors because the dark current caused the preamplifier to be overloaded.

Similar to the VLWIR detectors, the bias dependence of the response at high temperatures is an indicator of inefficiencies in the carrier transport process, which could be ascribed to the decreased diffusion length. Switching of the residual doping from $p$-type to $n$-type for InAs/GaSb SL material has been reported [14, 16-17]. Studies of mid-wave infrared InAs/GaSb SLs confirm that the material becomes $n$-type for temperatures higher than $120 \mathrm{~K}$ [17]. There is a strong possibility for a carrier-type alteration to occur at lower temperatures for LWIR photodetectors due to thicker layers of InAs that are included in each SL period. The resulting lower diffusion length of holes and the design modification in the growth order of these ICIP structures could worsen the carrier collection.

The normalized detectivity for R120 is displayed in Fig. 7. In contrast to the response curves that have their maximum at relatively significant bias at high temperatures, here $D^{*}$ did not increase with reverse bias. We also note that although the device resistance was higher at a small 
reverse bias compared to the zero-bias resistance, the increased dark current under a reverse bias had a more significant effect, resulting in a reduced value of $D^{*}$. In other words, the increased dark current at a reverse bias had more influence on the signal-to-noise ratio than the photoresponse increase and higher $R A$. At $78 \mathrm{~K}$, the highest $D^{*}$ for $\mathrm{R} 120$ and $\mathrm{R} 121$ at $\lambda=8.0 \mu \mathrm{m}$ under zero-bias operation was $4.4 \times 10^{10}$ and $4.5 \times 10^{10} \mathrm{~cm} \cdot \mathrm{Hz}^{1 / 2} / \mathrm{W}$, respectively.

\section{CONCLUDING REMARKS}

We have reported recent studies on interband cascade infrared photodetectors with VLWIR and LWIR cutoff wavelengths. Measurements for the VLWIR ICIPs show some non-idealities in the carrier transport that may arise from the presence of an unintentional electrostatic barrier and the $n$-type background doping of the SL absorber. The latter effect leads to lower collection efficiencies due to the inefficiency of hole transport in SL absorbers. We speculate that absorbers in these VLWIR and LWIR ICIPs are $n$-type, especially at high temperatures. In this case, holes would become the minority carriers with less efficient transport compared to the electrons. This is consistent with the observation that external bias was required to aid the collection of photocarriers. We have also observed high levels of dark current, which arise from a very narrow bandgap, high defect densities, and possible fabrication process issues. Our study on two-stage LWIR detectors shows improved device fabrication and material quality with lower defect densities, which resulted in the observed reduction in dark current density. Detectors fabricated from R120 exhibited bias dependent behavior only at high temperatures (167 K and above), providing additional evidence of inefficient carrier transport at high temperatures. The activation energies obtained from the temperature dependence of the dark current imply that the device was limited neither by diffusion nor G-R processes. We speculate that this dark current may arise from the partial doping architecture that is included in the absorber regions. The 
finding that hole transport influences the VLWIR detector performance indicates that modifications in the ICIP structures are needed. Such modifications may include: shorter absorbers and better band-edge alignments between the absorbers and the unipolar barriers. These modifications will be investigated in the future.

\section{ACKNOWLEDGMENTS}

This work was supported by NSF (Award Number ECCS-1202318), and by C-SPIN, the Oklahoma/Arkansas MRSEC (NSF Award Number DMR-0520550).

\section{REFERENCES}

[1] C. H. Grein, P. M. Young, and H. Ehrenreich, "Minority carrier lifetimes in ideal InGaSb/InAs superlattices," Appl. Phys. Lett., vol. 61, no. 24, p. 2905, 1992.

[2] C. H. Grein, P. M. Young, M. E. Flatté, and H. Ehrenreich, "Long wavelength InAs/InGaSb infrared detectors: Optimization of carrier lifetimes," J. Appl. Phys., vol. 78, no. 12, p. 7143, 1995.

[3] W. E. Tennant, “'Rule 07' Revisited: Still a Good Heuristic Predictor of p/n HgCdTe Photodiode Performance?," J. Electron. Mater., vol. 39, no. 7, pp. 1030-1035, Feb. 2010.

[4] D. R. Rhiger, "Performance Comparison of Long-Wavelength Infrared Type II Superlattice Devices with HgCdTe," J. Electron. Mater., vol. 40, no. 8, pp. 1815-1822, May 2011.

[5] M. Razeghi, a. Haddadi, a. M. Hoang, E. K. Huang, G. Chen, S. Bogdanov, S. R. Darvish, F. Callewaert, and R. McClintock, "Advances in antimonide-based Type-II superlattices for infrared detection and imaging at center for quantum devices," Infrared Phys. Technol., vol. 59, pp. 41-52, Jul. 2013.

[6] J. V. Li, R. Q. Yang, C. J. Hill, and S. L. Chuang, "Interband cascade detectors with room temperature photovoltaic operation," Appl. Phys. Lett., vol. 86, no. 10, p. 101102, 2005.

[7] R. Q. Yang, Z. Tian, J. F. Klem, T. D. Mishima, M. B. Santos, and M. B. Johnson, "Interband cascade photovoltaic devices," Appl. Phys. Lett., vol. 96, no. 6, p. 063504, 2010.

[8] R. Q. Yang, Z. Tian, Z. Cai, J. F. Klem, M. B. Johnson, and H. C. Liu, "Interbandcascade infrared photodetectors with superlattice absorbers," J. Appl. Phys., vol. 107, no. 5, p. 054514, 2010. 
[9] Z. Tian, R. T. Hinkey, R. Q. Yang, D. Lubyshev, Y. Qiu, J. M. Fastenau, W. K. Liu, and M. B. Johnson, "Interband cascade infrared photodetectors with enhanced electron barriers and p-type superlattice absorbers," J. Appl. Phys., vol. 111, no. 2, p. 024510, 2012.

[10] R. T. Hinkey, H. Lotfi, L. Li, H. Ye, L. Lei, R. Q. Yang, J. C. Keay, T. D. Mishima, M. B. Santos, and M. B. Johnson, "Interband cascade infrared photodetectors with $\mathrm{InAs} / \mathrm{GaSb}$ superlattice absorbers," Proc. SPIE vol. 8868, paper 886805, 2013.

[11] R. T. Hinkey and R. Q. Yang, "Theory of multiple-stage interband photovoltaic devices and ultimate performance limit comparison of multiple-stage and single-stage interband infrared detectors," J. Appl. Phys., vol. 114, no. 10, p. 104506, 2013.

[12] N. Gautam, S. Myers, a. V. Barve, B. Klein, E. P. Smith, D. R. Rhiger, L. R. Dawson, and S. Krishna, "High operating temperature interband cascade midwave infrared detector based on type-II InAs/GaSb strained layer superlattice," Appl. Phys. Lett., vol. 101, no. 2, p. 021106, 2012.

[13] R. Q. Yang, H. Lotfi, L. Li, R. T. Hinkey, H. Ye, J. F. Klem, L. Lei; T. D. Mishima, J. C. Keay, M. B. Santos, M. B. Johnson, "Quantum-engineered interband cascade photovoltaic devices” Proc. SPIE. 8993, p. 899310, 2014.

[14] A. Khoshakhlagh, F. Jaeckel, C. Hains, J. B. Rodriguez, L. R. Dawson, K. Malloy, and S. Krishna, "Background carrier concentration in midwave and longwave InAs/GaSb type II superlattices on GaAs substrate," Appl. Phys. Lett., vol. 97, no. 5, p. 051109, 2010.

[15] B.-M. Nguyen, S. Bogdanov, S. A. Pour, and M. Razeghi, "Minority electron unipolar phtodetectors based on type II InAs/GaSb/AlSb superlattices for very long wavelength infrared detection," Appl. Phys. Lett., vol. 95, no. 18, p. 183502, 2009.

[16] L. Bürkle, F. Fuchs, J. Schmitz, and W. Pletschen, "Control of the residual doping of InAs/(GaIn)Sb infrared superlattices," Appl. Phys. Lett., vol. 77, no. 11, p. 1659, 2000.

[17] C. Cervera, J. B. Rodriguez, J. P. Perez, H. Aït-Kaci, R. Chaghi, L. Konczewicz, S. Contreras, and P. Christol, "Unambiguous determination of carrier concentration and mobility for InAs/GaSb superlattice photodiode optimization," J. Appl. Phys., vol. 106, no. 3, p. 033709, 2009. 


\section{Figure captions}

Fig. 1 Dark current density vs. bias voltage for one-, two- and three-stage VLWIR detectors in a temperature range of 78-143 K.

Fig. 2 Responsivity spectra $\left(\mathrm{R}_{\lambda}\right)$ for a two-stage VLWIR detector at 78 and $100 \mathrm{~K}$ under reverse bias. Indicated voltages are the bias at which the maximum response was acquired. Inset (a): zero-bias $\mathrm{R}_{\lambda}$ at $\lambda=10 \mu \mathrm{m}$ for one, two- and three stage detectors with an increasing temperature. Inset (b): Zero-bias $\mathrm{R}_{\lambda}$ at 125 and $143 \mathrm{~K}$ for the two-stage VLWIR detector.

Fig. 3 Detectivity $D^{*}$ for representative one-, two- and three-stage VLWIR ICIPs at $78 \mathrm{~K}$.

Fig. 4 Dark current density at 78 K for two devices made from R120 and R121, respectively.

Fig. 5 Dark current density at different temperatures for a LWIR detector from R120. The inset shows the fitted activation energy for the Arrhenius plot of the dark current.

Fig. 6 Responsivity spectra of a detector from R120 at temperatures up to $220 \mathrm{~K}$. Inset shows its zero-bias responsivity spectra at 240 and $250 \mathrm{~K}$.

Fig. 7 Detectivity $D^{*}$ for a detector made from R120 at temperatures up to $220 \mathrm{~K}$. 
Fig. 1
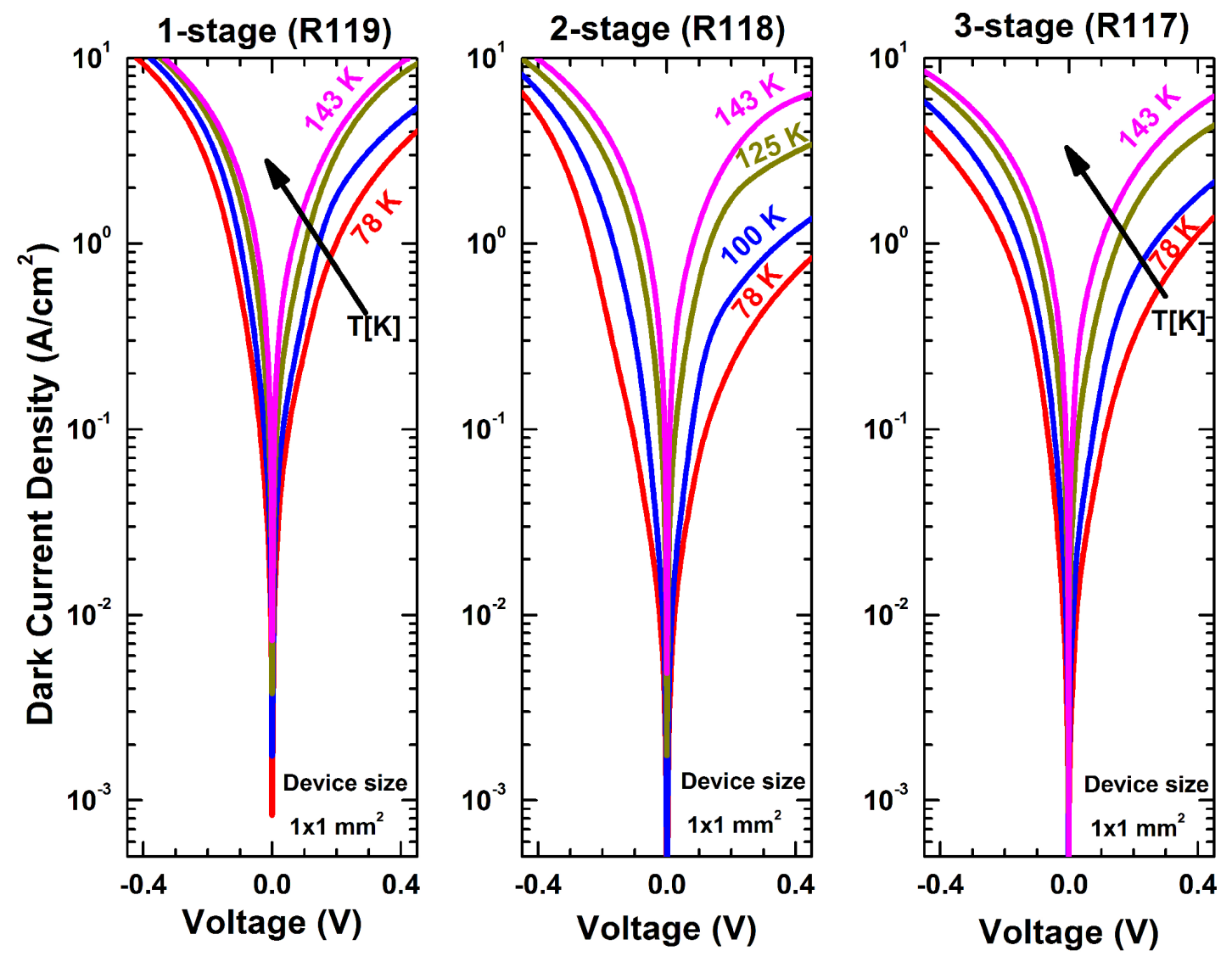
Fig. 2

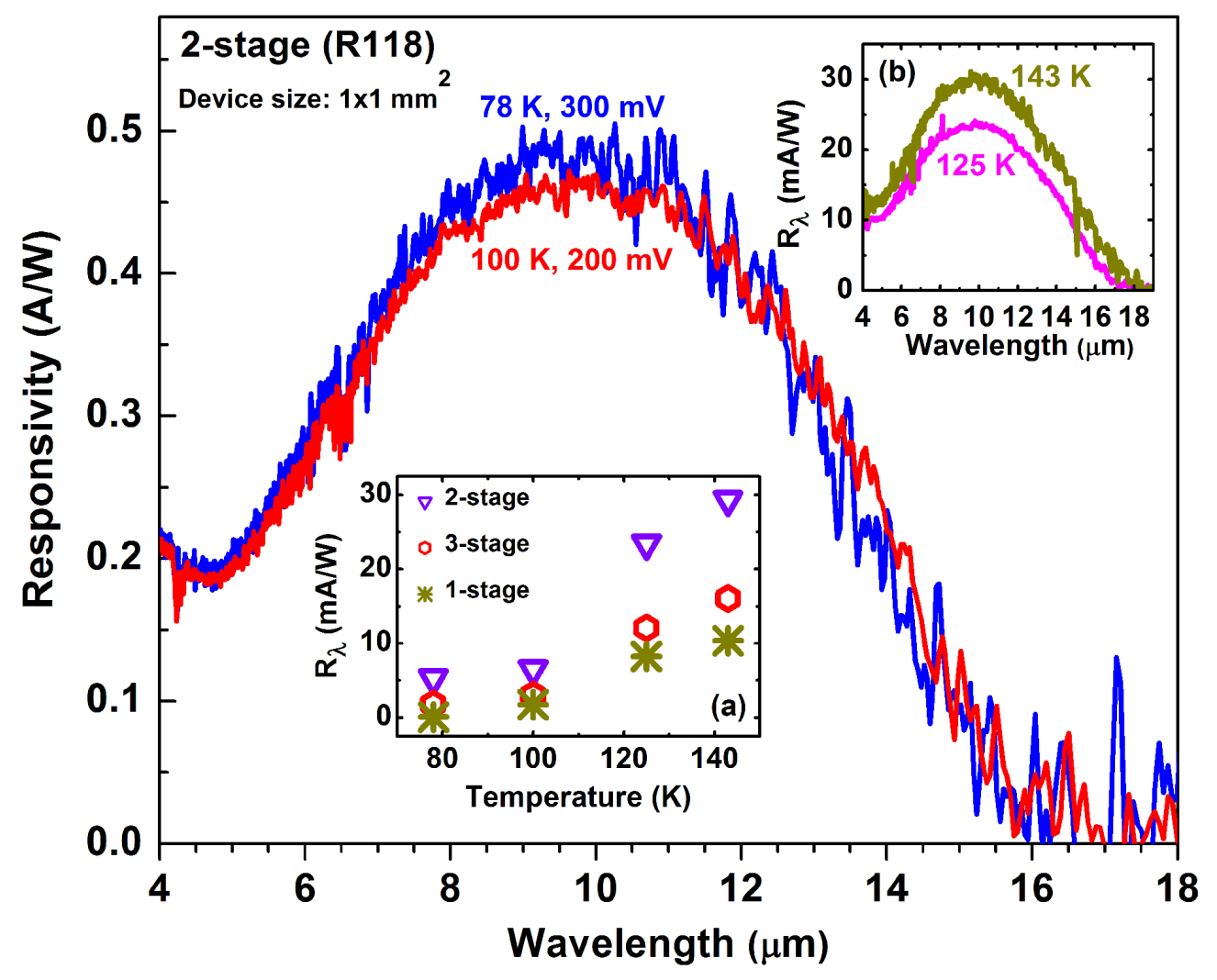


Fig. 3

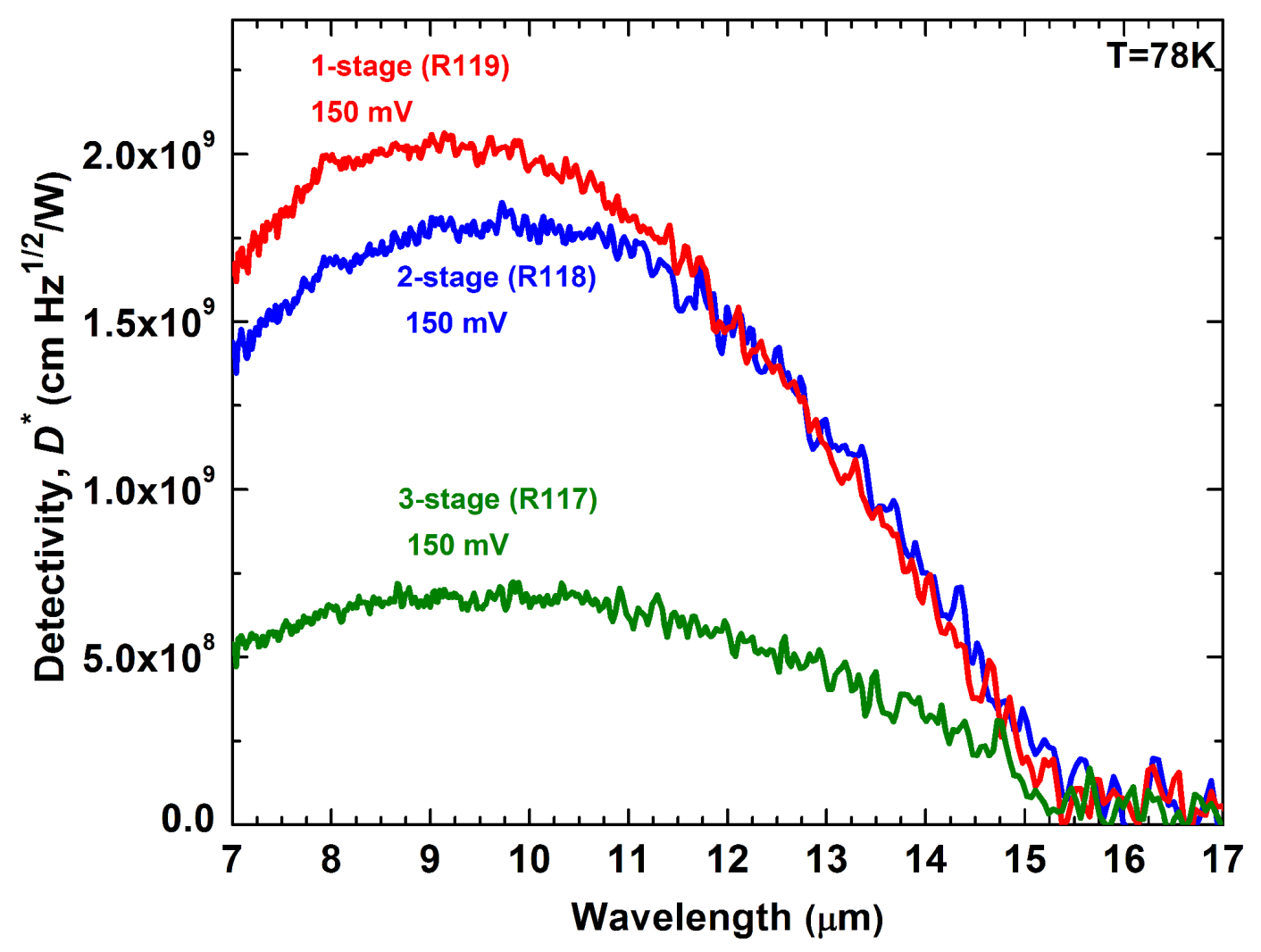


Fig. 4

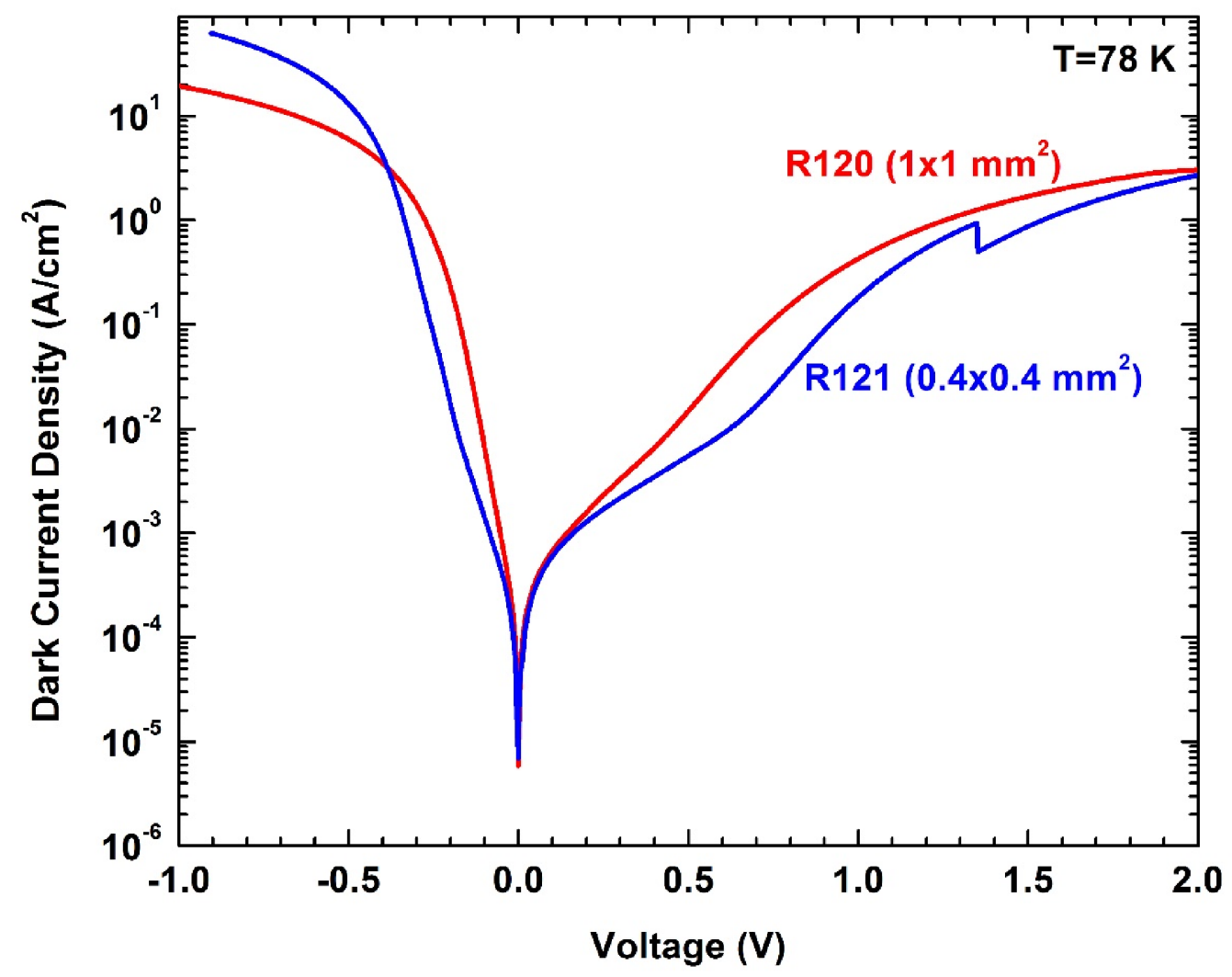


Fig. 5

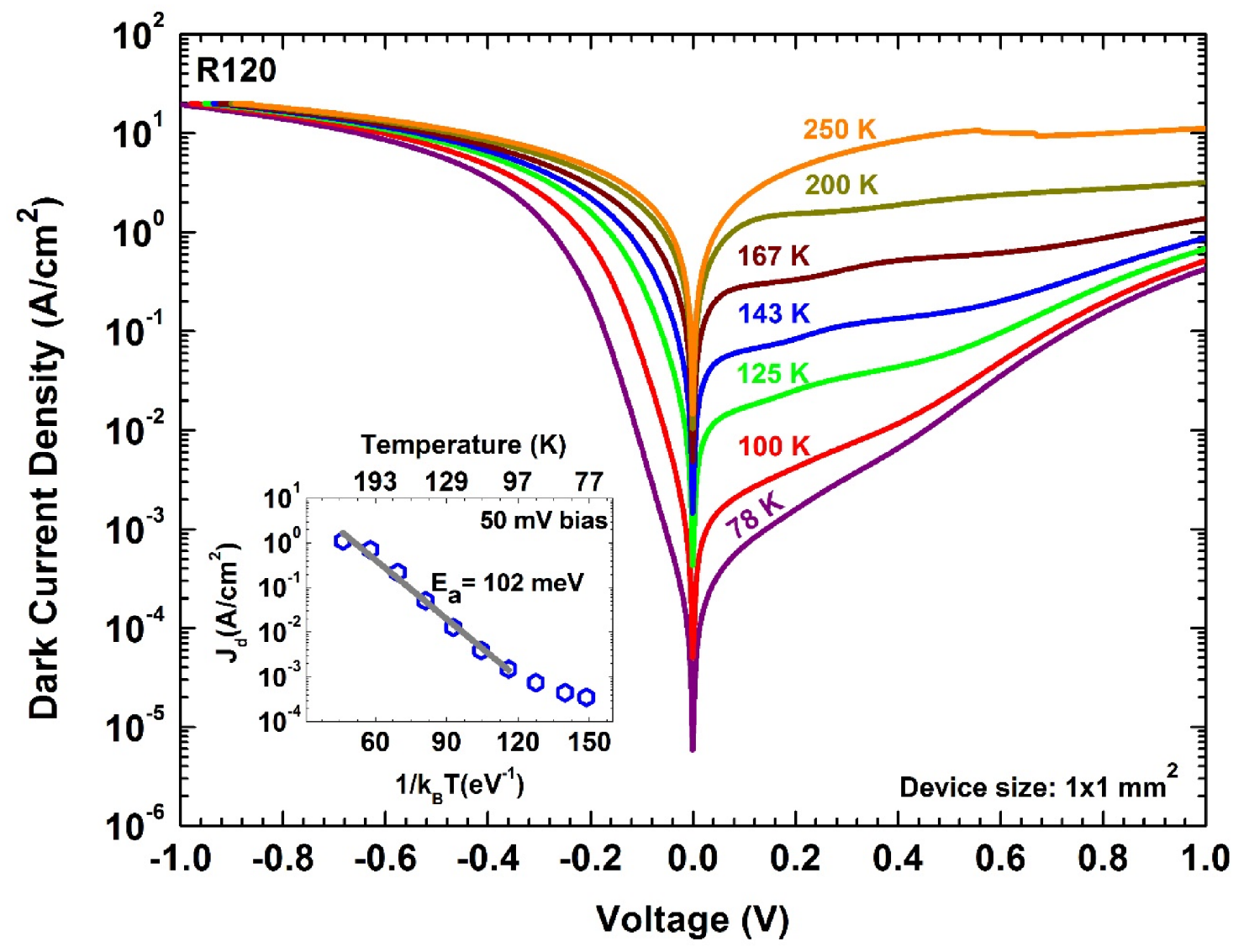


Fig. 6

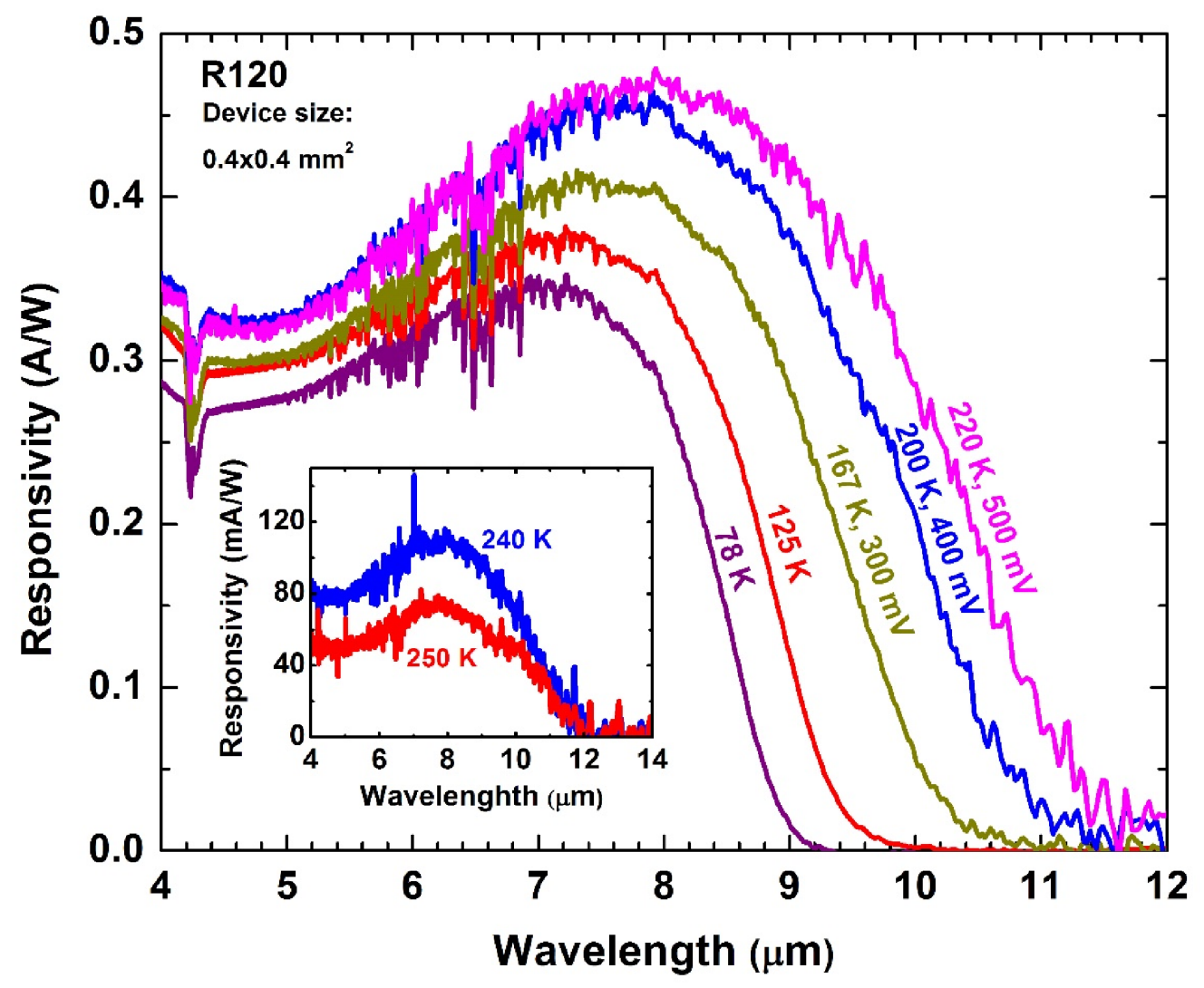


Fig. 7

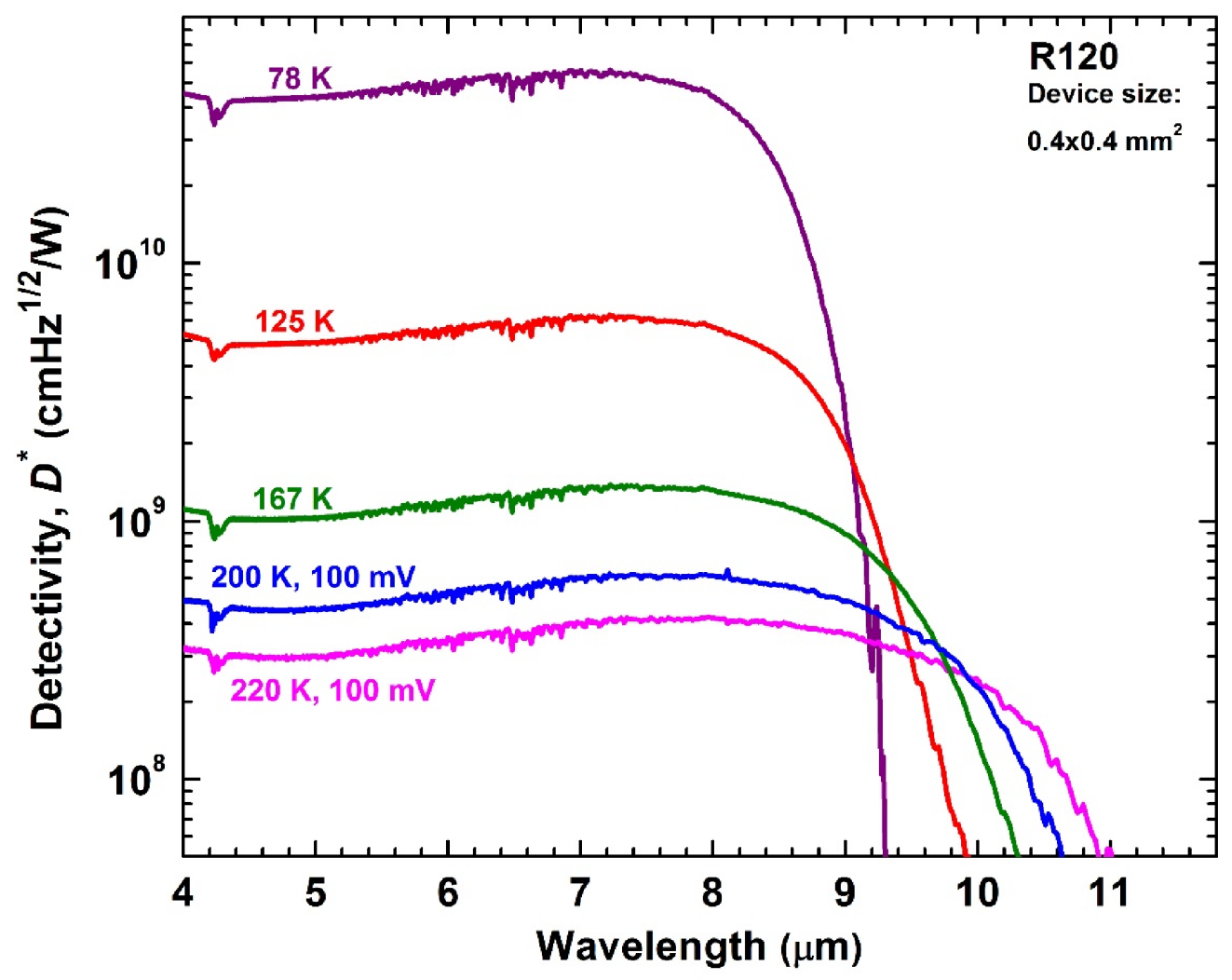

